\title{
Zinc Ferrite Nanoparticles via Coprecipitation Modified Method: Glycerol as Structure Directing and Stabilizing Agent
}

\author{
Edilan S. Lima, ${ }^{a}$ Luelc S. Costa, ${ }^{b}$ Gabrielle R. L. M. Sampaio, ${ }^{c}$ Edipo S. Oliveira, ${ }^{c}$ \\ Erandir B. Silva, ${ }^{c}$ Hélio O. Nascimento, ${ }^{d}$ Ronaldo F. Nascimento, ${ }^{\circledR d}$ Karine O. Moura ${ }^{e}$ \\ Moisés Bastos-Neto, ${ }^{e}$ Adonay R. Loiola ${ }^{\circledR *}$ and José M. Sasakif \\ ${ }^{a}$ Departamento de Engenharia de Metalúrgica e de Materiais, Universidade Federal do Ceará, \\ Campus do Pici, 60440-900 Fortaleza-CE, Brazil \\ ${ }^{b}$ Laboratório Nacional de Nanotecnologia (LNNano), \\ Centro Nacional de Pesquisa em Energia e Materiais (CNPEM), 13083-970 Campinas-SP, Brazil \\ 'Departamento de Química Orgânica e Inorgânica, Universidade Federal do Ceará, \\ Campus do Pici, 60440-900 Fortaleza-CE, Brazil \\ ${ }^{d}$ Departamento de Química Analítica e Físico-Química, Universidade Federal do Ceará, \\ Campus do Pici, 60440-900 Fortaleza-CE, Brazil \\ ${ }^{e}$ Departamento de Engenharia Química, Universidade Federal do Ceará, Campus do Pici, \\ 60440-900 Fortaleza-CE, Brazil \\ ${ }^{f}$ Departamento de Física, Universidade Federal do Ceará, Campus do Pici, \\ 60440-900 Fortaleza-CE, Brazil
}

\begin{abstract}
Coprecipitation is one of the most practical methods used to synthesize ferrite nanoparticles. Fine characteristics of these materials can be improved by means of a series of modifications in the synthesis method. In this paper we present a study of influence of glycerol as structure directing and stabilizing agent in the synthesis of zinc ferrite nanoparticle, exploring its chelating capacity and oxidation. The studied materials include two series of zinc ferrite samples and its precursors obtained with or without glycerol throughout different stages during the synthesis process. The structural and morphological characteristics were evaluated by means of different techniques such as X-ray diffraction, Fourier transformed infrared spectroscopy, scanning and transmission electron microscopy and thermal analysis. The oxidation of glycerol was determined by high-performance liquid chromatograph. The obtained results demonstrate that zinc ferrite crystals synthesized in the presence of glycerol are initially bigger than those synthesized in absence of it, but according to the oxidation process they tend to decrease giving rise to intermediate phases. Interestingly, these samples grow back during more advanced stages and become structurally better organized, compared to the series of samples produced in absence of glycerol. These results indicate that glycerol is capable to modify the synthesis route of zinc ferrite nanoparticles via coprecipitation, acting directly over the size and morphology of the crystals.
\end{abstract}

Keywords: ferrites, nanoparticles, crystals/crystallization, spinels, glycerol

\section{Introduction}

Spinel ferrites have received increased attention in fundamental research due to their electrical, magnetic, optical and catalytic properties, finding applications in a variety of industrial sectors. ${ }^{1-3}$ Among the spinel system,

*e-mail: adonay@ufc.br zinc ferrite $\left(\mathrm{ZnFe}_{2} \mathrm{O}_{4}\right)$ is found to be one of the most extensively studied. It possesses an $\mathrm{AB}_{2} \mathrm{O}_{4}$ structure with tetrahedral A site occupied by $\mathrm{Zn}^{2+}$ ions and octahedral B site with $\mathrm{Fe}^{3+}$ ions in a face-centered cubic unit cell. ${ }^{4}$ This ceramic presents high electromagnetic performance, ${ }^{1,5}$ great chemical stability ${ }^{3,6}$ and good catalytic activity. ${ }^{6,7}$ Such properties are strongly influenced by particle size, agglomeration and morphology, ${ }^{8,9}$ which can be controlled 
in the synthesis process. The correlation between synthesis parameters and resulting physical properties provides insights on the activity and stability of these spinel ferrites. Therefore, several studies have been performed using a variety of wet chemistry techniques, such as sol-gel, ${ }^{10,11}$ hydrothermal, ${ }^{12-14}$ polymeric precursor method, ${ }^{15,16}$ sol-gel auto-combustion, ${ }^{17,18}$ polyol $^{19}$ and coprecipitation. ${ }^{20-24}$

Coprecipitation is one of the most widely used methods for the synthesis of ferrite nanomaterials. ${ }^{25,26}$ This method consists of mixing aqueous solutions of metal salts at certain molar ratios in highly basic solutions, either at room temperature or at elevated temperature. The nanoparticles morphology and size depend on the type of salt used, ionic strength, $\mathrm{pH}$ and other reaction parameters such as stirring rate, dropping speed of basic solution, etc. ${ }^{26,27}$ However, the main challenge in this approach lies on the control of particles aggregation, once strong dipole-dipole magnetic interactions lead to a wide distribution in crystal sizes and coalescence effects caused by the thermal treatment can significantly affect the material efficiency. In principle, surfactant/polymer or ionic groups have been used to increase the stability and dispersion of nanoparticles, preventing the agglomeration and coalescence phenomena. ${ }^{25,28-30} \mathrm{In}$ this context, glycerol has been reported as an excellent chelating agent, once the ability of alcohol adsorption to prevent excessive growth and hydrophilic hydroxyl groups on the surface of the produced nanoparticles gives greater stability and avoid aggregation. ${ }^{31}$ Furthermore, glycerol is an organic compound widely used in single-component reactions such as oxidation and hydrogenolysis due to the susceptibility of three hydroxyl groups in the glycerol molecule. ${ }^{32}$

In this work, we investigated an alternative coprecipitation route using glycerol for the production of zinc ferrites. In this route, we explore the chelating capacity of the glycerol, combined with its oxidation and the generated products so that structural and morphological aspects of the zinc ferrites nanoparticles are achieved. The structural and morphological characteristics of the nanoparticles were evaluated by means of different techniques such as X-ray diffraction (XRD), Fourier transformed infrared spectroscopy (FTIR), scanning (SEM) and transmission electron microscopy (TEM) and thermal analysis. The oxidation products of glycerol were determined by high-performance liquid chromatography (HPLC). The obtained results demonstrate that zinc ferrite crystals synthesized in the presence of glycerol are initially bigger than those synthesized in absence of it, but according to the oxidation process they tend to decrease giving rise to intermediate phases. Interestingly, these samples grow again during further stages and become structurally better organized, compared to the series of samples produced in absence of glycerol.

\section{Experimental}

Synthesis

All chemicals used, $\mathrm{FeCl}_{3} \cdot 6 \mathrm{H}_{2} \mathrm{O}, \mathrm{ZnCl}_{2}, \mathrm{NaOH}$ and glycerol were of analytical grade, purchased from SigmaAldrich and used as received. Two series of zinc ferrite samples have been prepared by coprecipitation methods. The first series consists of the modified coprecipitation method, in which the samples were prepared with glycerol as structure directing and stabilizing agent. The samples prepared include intermediates that were characterized so that the role played by glycerol could be studied. For the preparation of these samples a mixed aqueous solution was prepared by dissolving $3.3637 \mathrm{~g}$ of $\mathrm{FeCl}_{3} \cdot 6 \mathrm{H}_{2} \mathrm{O}$ and $0.8481 \mathrm{~g}$ of $\mathrm{ZnCl}_{2}$ in $3 \mathrm{~mL}$ of distilled water. Glycerol was added to this solution (4 mL), under vigorous stirring, and then $10 \mathrm{~mL}$ of $4.97 \mathrm{~mol} \mathrm{~L}^{-1} \mathrm{NaOH}$ (precipitating agent) was added dropwise. An aliquot of the formed suspension was centrifuged and the separated solid named as $\mathrm{S} 1$-gly (stage 1). The main suspension was transferred to an alumina tube and heated to $150{ }^{\circ} \mathrm{C}$ for $15 \mathrm{~h}$, under static conditions. An aliquot of the resulting material was separated, centrifuged and the solid named as S2-gly (stage 2). The suspension was then heated at $350{ }^{\circ} \mathrm{C}$ for $1 \mathrm{~h}$ under rotation in air atmosphere $\left(90 \mathrm{~mL} \mathrm{~min}^{-1}\right)$. Again, an aliquot was separated, and named as S3-gly (stage 3). The remaining material was heated at $600{ }^{\circ} \mathrm{C}$, under the same atmosphere and rotation conditions of the previous sample. The obtained material was named as S4-gly (stage 4). All these samples were washed with distilled water, centrifuged at $6000 \mathrm{rpm}$ and dried at $100{ }^{\circ} \mathrm{C}$ for $8 \mathrm{~h}$.

The second series consists of zinc ferrite nanoparticles synthesized by means of the traditional coprecipitation method. In this case, the synthesis procedures were similar to the above described method, but without using glycerol. The correspondent obtained samples were named as S1- $\varnothing$, $\mathrm{S} 2-\varnothing, \mathrm{S} 3-\varnothing$ and $\mathrm{S} 4-\varnothing$. For clarity, the synthesis process is schematically summarized in Figure 1.

\section{Characterization}

XRD experiments were performed using an X-ray powder diffractometer Xpert MPD (Panalytical). X-ray tube (Co) operated at $40 \mathrm{kV}$ and $40 \mathrm{~mA}$. The high-resolution diffraction is obtained with a hybrid monochromator for incidence beam, which consists of mirror and Ge monochromator producing a parallel and highly 


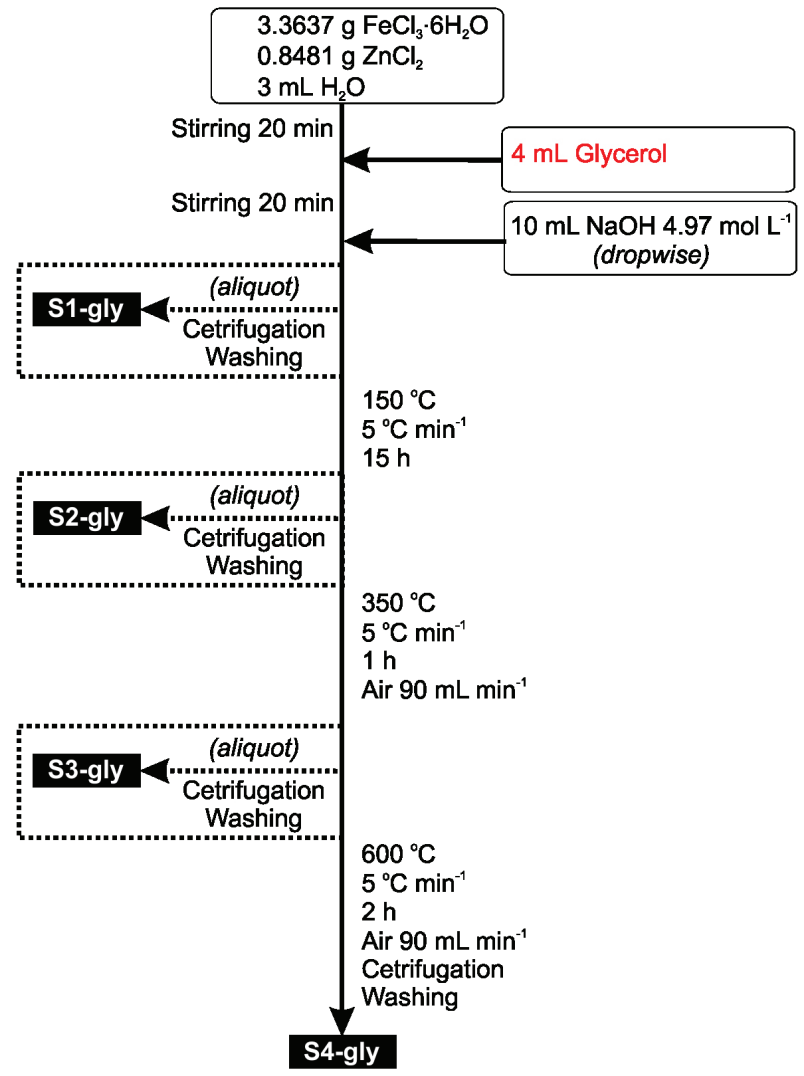

Figure 1. Flowchart of the synthesis protocol for the production of zinc ferrite by coprecipitation method. The modified coprecipitation method is characterized by the addition of glycerol (in red, upper right) after the dissolution of iron and zinc salts.

monochromatic beam, respectively. The data were collected with Pixcel solid-state detector. The XRD measurements were performed by using a 255 channels detector, with step scan of $0.013^{\circ}$ in $2 \theta$ angular interval from $10^{\circ}$ to $100^{\circ}$, with counting time of $150 \mathrm{~s}$. Structure refinement and quantitative analysis of the obtained nanoparticles were carried out by the Rietveld method using GSAS software package with EXPGUI interface ${ }^{33,34}$ (see Figures $\mathrm{S} 1$ and S2 in the Supplementary Information (SI) section). The crystallographic data of zinc ferrite $\left(\mathrm{ZnFe}_{2} \mathrm{O}_{4}\right)$, dihydrate zinc oxalate $\left(\mathrm{ZnC}_{2} \mathrm{O}_{4} \cdot 2 \mathrm{H}_{2} \mathrm{O}\right)$ and zinc oxide $(\mathrm{ZnO})$ were imported from the inorganic crystal structure database (ICSD): 85866, 56466 and 76641, respectively. ${ }^{35-37}$ These results were used to calculate the average crystallite size of the samples through Scherrer equation, ${ }^{38} \mathrm{D}=(\mathrm{k} \lambda) /(\beta \cos \theta)$, where $\mathrm{k}$ is the shape coefficient, $\theta$ is the Bragg angle and $\beta$ is the full-width at half maximum (FWHM) of the diffraction peaks, which was corrected for instrumental broadening.

FTIR spectra were obtained by using a Shimadzu IRealise FTIR spectrometer in the $4000-400 \mathrm{~cm}^{-1}$ region, with nominal resolution of $2 \mathrm{~cm}^{-1}$. For these experiments, the samples were prepared in $\mathrm{KBr}$ wafers.
SEM analyses were carried out in FEG-FEI equipment model Quanta 450. The nonmagnetic samples were prepared on double-sided carbon tape on an aluminum support. For the magnetic samples, colloidal silver paste was used instead of double-sided carbon tape. All the samples were coated with a thin layer of gold.

TEM analyses were performed by using a few milligrams of the samples, in the powder form, dispersed in isopropyl alcohol and sonicated for $15 \mathrm{~min}$. Two drops of the dispersion were placed onto ultra-thin carbon coated copper grids. After deposition, the samples were dried at room temperature overnight prior to the obtainment of the images. The images were acquired using a JEOL JEM 2100 $\mathrm{LaB}_{6}$ operating at an accelerating voltage equal to $200 \mathrm{kV}$ and equipped with a TV (Gatan ES500 W). High-resolution TEM (HRTEM), high angle annular dark-field scanning transmission electron microscopy (HAADF-STEM), and STEM energy-dispersive spectroscopy (STEM-EDS) analyses were obtained using a JEOL 2010F field emission microscope operating at $200 \mathrm{kV}$ and equipped with an $\mathrm{X}$-MaxN $80 \mathrm{~T}$ detector of Oxford Instrument. AZtecTEM EDS microanalysis software was used for EDS data analyses. Particle distributions were determined using Image J software. ${ }^{39}$

Thermogravimetric analyses (TGA) were carried out using a TGA/QMS STA 409 CD/403/5/G SKIMMER model (Netzsch). The experimental procedure consisted of weighing approximately $20 \mathrm{mg}$ of each sample in alumina crucibles using a heating rate of $5{ }^{\circ} \mathrm{C} \mathrm{min}^{-1}$, from ca. 30 to $900{ }^{\circ} \mathrm{C}$, under air $\left(\mathrm{N}_{2} / \mathrm{O}_{2}\right)$ flow rate of $50 \mathrm{~mL} \mathrm{~min}^{-1}$.

HPLC analyses were performed using a Shimadzu LC20AT with diode array detector (DAD) and detection wavelength was set at $210 \mathrm{~nm}$. Chromatographic separation was performed with an AMINEX HPX-87H column $(300 \times 7.8 \mathrm{~mm})$ with $\mathrm{H}_{2} \mathrm{SO}_{4}$ solution as the mobile phase $\left(3 \mathrm{mmol} \mathrm{L}^{-1}\right)$ with flow rate of $0.4 \mathrm{~mL} \mathrm{~min}^{-1}$, in an isocratic mode at $60{ }^{\circ} \mathrm{C}$. The injection volume was fixed at $20 \mu \mathrm{L}$ for all samples. The HPLC method was based on previously published method..$^{40}$ The samples S1-gly and S2-gly were washed with $50 \mathrm{~mL}$ mobile phase and centrifuged for $7 \mathrm{~min}$ at $7500 \mathrm{rpm}$ and then their supernatant were injected onto HPLC system. Glyceraldehyde (2,3-dihydroxypropanal), glyceric acid (2,3-dihydroxypropanoic acid), dihydroxyacetone (1,3-dihydroxypropan-2-one), tartronic acid (2-hydroxypropanedioic acid) and glycolic acid (2-hydroxyethanoic acid) were obtained from SigmaAldrich and used as standards. In addition, the oxalic acid (ethanedioic acid) and formic acid (methanoic acid) were also used as chemical standards and were purchased from Dinâmica. The chromatograms of the standards are shown in Figure S8 (SI section). 


\section{Results and Discussion}

X-ray diffraction (XRD)

Figures 2 and 3 show the results for the two series of samples prepared according to the modified and the traditional methods, respectively. In addition, Rietveld refinement results and quantitative analyses of all obtained nanoparticles are presented in Figures S1, S2, Tables S1 and S2 (SI section). By using both methods, the diffraction patterns obtained for the samples at the stage 1 (Figures $2 b$ and $3 \mathrm{~b}$ ) show the presence of only a zinc ferrite crystalline phase. The insets of Figures 2 and 3 show the diffraction peaks with amplified intensity, which provide an easier visualization. Moreover, the sample produced in presence of glycerol presents FWHM lower than for the sample obtained via traditional route, which indicates that there is difference in the crystallite sizes depending on the method used. In fact, the average particles size calculated by Scherrer equation is $5 \mathrm{~nm}$ for the sample S1-gly and $2 \mathrm{~nm}$ for the sample S1- $\varnothing$. The bigger crystal size observed for the sample S1-gly can be considered a direct result of the variation in the precursor salts solubility in the presence of glycerol, due to the hydrogen bonding between hydroxyl groups of glycerol and the ions in the solution. ${ }^{41}$ Despite this, both samples (S1-gly and S1- $\varnothing$ ) are composed of crystalline materials with very small dimensions. In principle, this reduced crystal sizes are associated with basicity and the ionic strength of the precipitation medium, i.e., the higher the $\mathrm{pH}$ the smaller the crystal size. ${ }^{42,43}$

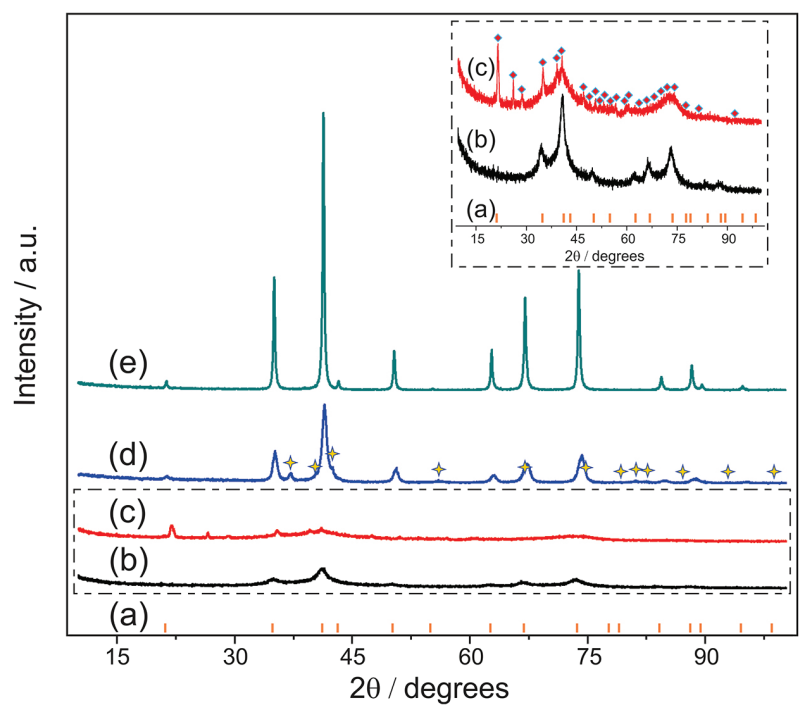

Figure 2. XRD patterns of the samples obtained by the modified method: (a) standards for zinc ferrite peaks; (b) S1-gly; (c) S2-gly; (d) S3-gly (with the highlighted peaks referring to zinc oxide phase) and (e) S4-gly. The inset shows (a) standards for zinc oxide peaks and, in a magnified scale, (b) S1-gly and (c) S2-gly (with the highlighted peaks referring to zinc oxalate phase).

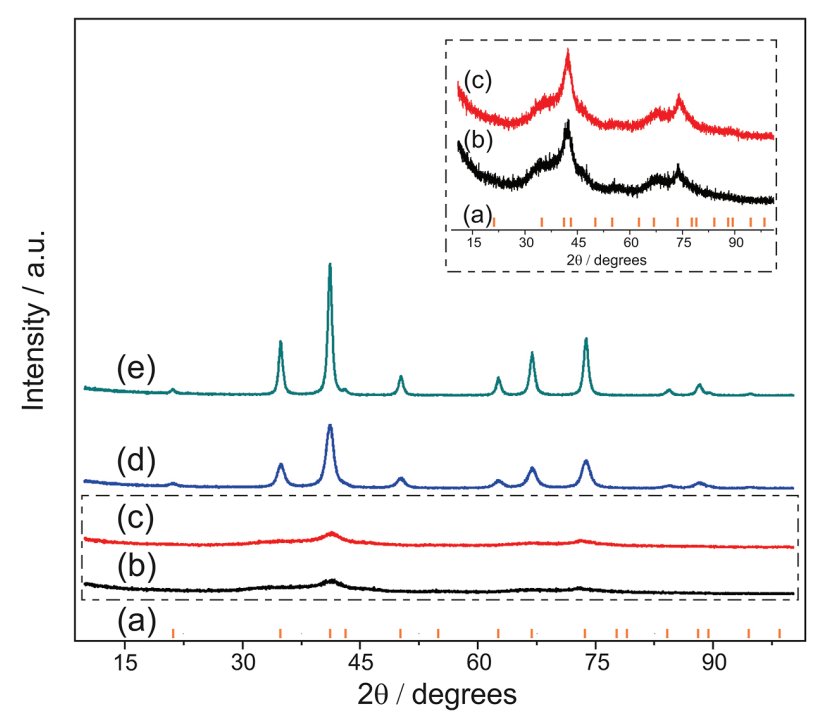

Figure 3. XRD patterns of the samples obtained by the traditional method: (a) standards for zinc ferrite peaks; (b) S1- $\varnothing$; (c) S2- $\varnothing$; (d) S3- $\varnothing$ and (e) S4- $\varnothing$. The inset shows (a) standards for zinc oxide peaks and, in a magnified scale, (b) S1- $\varnothing$ and (c) S2- $\varnothing$.

For the sample S2-gly (Figure 2c) two phases were identified, namely, zinc ferrite and dihydrate zinc oxalate. The average crystal size obtained by Scherrer equation to zinc ferrite is $2 \mathrm{~nm}$ and for dihydrate zinc oxalate is $10 \mathrm{~nm}$. It should be noted that ferrites can act as catalyst in the glycerol oxidation reactions. ${ }^{44,45}$ This oxidation reactions could potentially produce compounds such as glyceraldehyde and dihydroxyacetone (simultaneous and/or subsequent), which by further oxidation can be converted to some oxidized products as glyceric, tartronic, formic, glycolic and oxalic acid. ${ }^{44,46}$ The presence of dihydrate zinc oxalate at the end of stage 2 indicates the occurrence of this process. The oxalate salt observed would result from the dissolution reaction of the zinc ferrite by the oxalic acid formed in situ. ${ }^{47}$ From this perspective, a decrease in the average crystal size observed for the zinc ferrite phase (compared to the sample S1-gly) becomes clear. On the other hand, when sample $\mathrm{S} 2-\varnothing$ (Figure $3 \mathrm{c}$ ) is compared with the previous one $(\mathrm{S} 1-\varnothing)$, no difference in size is observed, revealing that the thermal treatment applied was not able to cause perceptive changes in the crystalline structure.

As shown in Figure 2d, two crystalline phases were identified for the sample S3-gly: zinc ferrite and zinc oxide, and their average crystal sizes were found to be 13 and $9 \mathrm{~nm}$, respectively. On the other hand, without the addition of glycerol (S3-Ø), zinc oxide formation did not occur and only zinc ferrite phase (crystal size of $10 \mathrm{~nm}$ ) was identified. According to the XRD data analysis, the zinc ferrite structure observed in both S3-gly and S3- $\varnothing$ samples presented similar characteristics. However, the growth of zinc ferrite nanoparticles in the sample S3-gly 
was accompanied by a thermal degradation event of the dihydrate zinc-oxalic phase, i.e, water and $\mathrm{CO}_{2}$ were released from this salt during the heating process, resulting in the zinc oxide phase. ${ }^{48,49}$ Note that the calculated quantity of zinc oxide phase in the sample differs from what is expected due to the degradation (see Table S1, SI section), which means that some zinc atoms might have been incorporated into the zinc ferrite structure.

The samples obtained at the end of stage 4 present XRD patterns of pure zinc ferrite nanoparticles with average crystal size of $46 \mathrm{~nm}$ for the sample S4-gly (Figure 2e) and $20 \mathrm{~nm}$ for the sample S4- $\varnothing$ (Figure 3e). These different sizes essentially highlight the role of glycerol as well as its derivatives in the overall crystallization process. For the modified method, the larger size of the precipitated crystal in the stage 1 implies in a small number of nucleated crystals. ${ }^{50}$ In stage 2 , this number of nucleated crystals is further reduced. The acid attack not only reduced the average size of the produced crystals, but also decreased the total amount, which means that a complete dissolution event might have occurred in smaller crystals. On the other hand, during the traditional method of co-precipitation, the amount of crystals remains unchanged during the similar stages. Those variations lead to different average size of zinc ferrite nanoparticles at the final stage (S4).

\section{FTIR}

Figures 4 and 5 show the FTIR spectra of samples prepared by using the modified method and the traditional one, respectively. The sample S1-gly (Figure 4a) presents bands at 1103 and $1047 \mathrm{~cm}^{-1}$, which are not observed for the sample $\mathrm{S} 1-\varnothing$ (Figure 5a). These bands are related to the presence of secondary alcohols, ${ }^{51}$ indicating the presence of glycerol. These bands are also observed for the sample S2-gly (Figure 4b), indicating that at least some of the initial glycerol is still preserved after the first thermal treatment, at $150{ }^{\circ} \mathrm{C}$. The band characteristic of $\mathrm{O}-\mathrm{H}$ bending vibration for adsorbed water is verified at $1627 \mathrm{~cm}^{-1}$ for the sample S2-gly and at $1619 \mathrm{~cm}^{-1}$ for the sample S2- $\varnothing$ (Figure 5b). ${ }^{52,53}$ This band presents higher intensity for the first sample, in which glycerol is present. The shift towards a higher frequency for the sample with glycerol may be due to stronger interactions.

The band at $1318 \mathrm{~cm}^{-1}$ observed for the sample S2-gly may be associated to symmetric $\mathrm{C}=\mathrm{O}$ bond vibrations, ${ }^{8,54}$ indicating the formation of glycerol oxidized products, which is in accordance with the XRD results for the production of zinc oxalate. According to the selection rules for the $\mathrm{O}_{\mathrm{h}}{ }^{7}$ space group, which represents normal spinel ferrite structures, four triple degenerated $t_{1 u}$ infrared

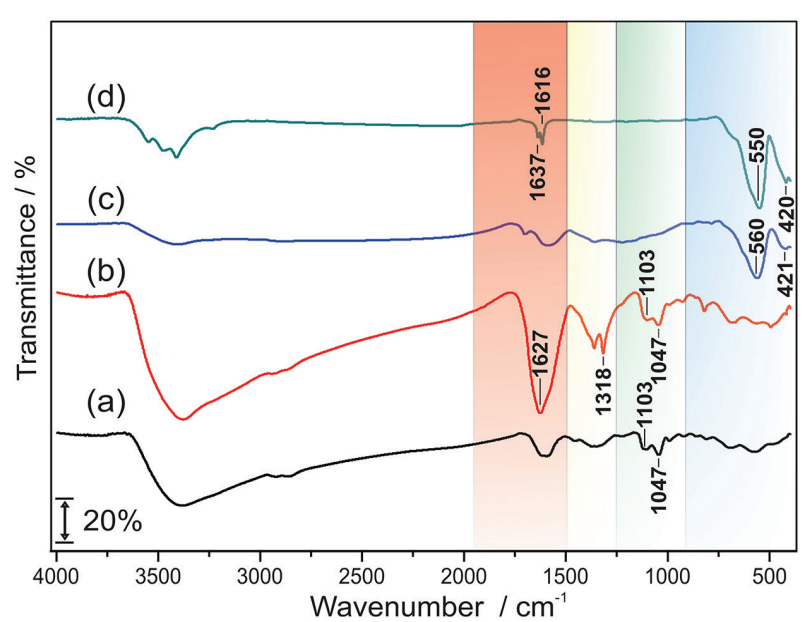

Figure 4. FTIR spectra of samples obtained with the presence of glycerol: (a) S1-gly; (b) S2-gly; (c) S3-gly; (d) S4-gly.

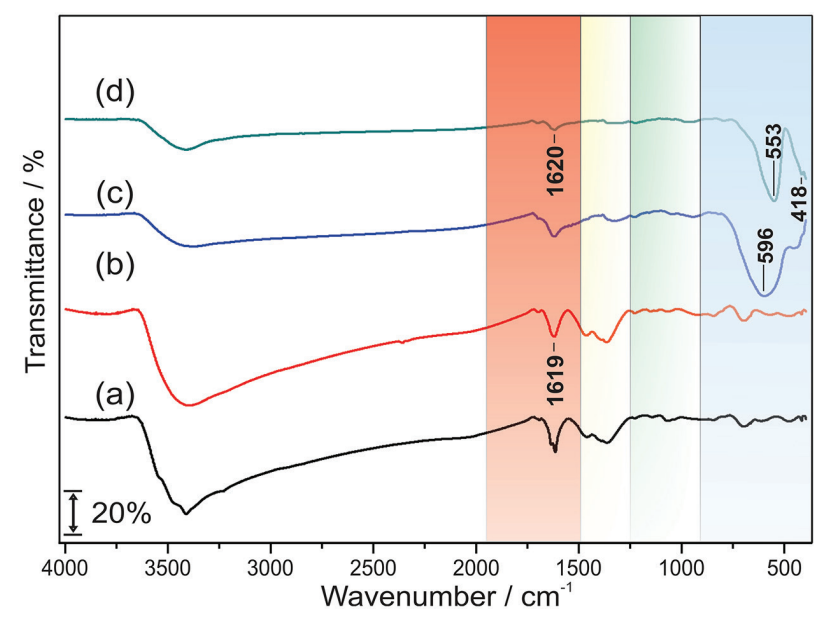

Figure 5. FTIR spectra of samples obtained without the presence of glycerol: (a) S1- Ø; (b) S2- Ø; (c) S3- ; (d) S4- Ø.

active modes are expected. ${ }^{55,56}$ For the sample S4-gly (Figure $4 \mathrm{~d}$ ), $v_{1}$ is observed at $550 \mathrm{~cm}^{-1}$ and $v_{2}$ at $420 \mathrm{~cm}^{-1}$. They correspond to intrinsic stretching vibration of $\mathrm{Zn}-\mathrm{O}$ bonds in tetrahedral sites and $\mathrm{Fe}-\mathrm{O}$ bonds in octahedral sites, respectively. ${ }^{8,15,53}$ The sample S4- $\varnothing$ (Figure 5d) presents these bands at 553 and $418 \mathrm{~cm}^{-1}$. A shoulder close to the $v_{1}$ and $v_{2}$ can be attributed to cations with different ionic states both in octahedral and in tetrahedral sites. ${ }^{57}$ The same bands are observed for samples S3-gly (Figure 4c) and S3- $\varnothing$ (Figure 5c), but shifted when compared to the corresponded S4 samples. The difference of $v_{1}$ between the samples S3-gly and S4-gly is only $10 \mathrm{~cm}^{-1}$ (560 and $550 \mathrm{~cm}^{-1}$ ), while for samples S3- $\varnothing$ and S4- $\varnothing$ the difference is $43 \mathrm{~cm}^{-1}$ (596 and $553 \mathrm{~cm}^{-1}$ ). A large shoulder in the band at $596 \mathrm{~cm}^{-1}$ for the sample $\mathrm{S} 3-\varnothing$ can be attributed to the presence of cations with different ionic states in both sites,${ }^{50}$ indicating that the sample prepared with glycerol presents a structure better organized before the final stage of calcination that the sample prepared without glycerol. 


\section{SEM and TEM}

According to the XRD results, glycerol and its derivatives lead to major transformations in the sample produced during stage S2. This way, Figure 6 shows details of the structure for S2-gly and S2- $\varnothing$ samples. HRTEM images display crystallographic patterns for both methods, confirmed by their fast Fourier transform (FFT) (inset of Figures $6 \mathrm{a}$ and $6 \mathrm{~b}$ ), which are in agreement with the zinc ferrite phase, corroborating with the XRD data.

Figure 6 a shows a resin-like material obtained from glycerol that encapsulates small crystals in the sample. This is due to the chelating capacity of glycerol and its oxidation process, which interfere in the nucleation and growth processes of the ferrite crystals formation, as can be seen in Figure S3 (SI section). On the other hand, the sample S2- $\varnothing$ presents crystals with different sizes regimes and uncontrolled aggregation, a behavior expected for the sample obtained by traditional method (see Figure S4, SI section).

The HAADF-STEM image and the corresponding quantitative EDS maps of the S2-gly sample (Figure 7) show isolated solids containing $\mathrm{Fe}$ and $\mathrm{Zn}$, and also other solids in which only $\mathrm{Zn}$ is detected. The presence of zinc-rich and iron-poor domains is in accordance with the expected products of glycerol oxidation and with the XRD results. In Figure S5 (SI section), another region can be visualized, possibly indicating that this behavior is common to the sample. Furthermore, amorphous solids containing $\mathrm{Fe}$ and $\mathrm{Zn}$ are also expected as a consequence
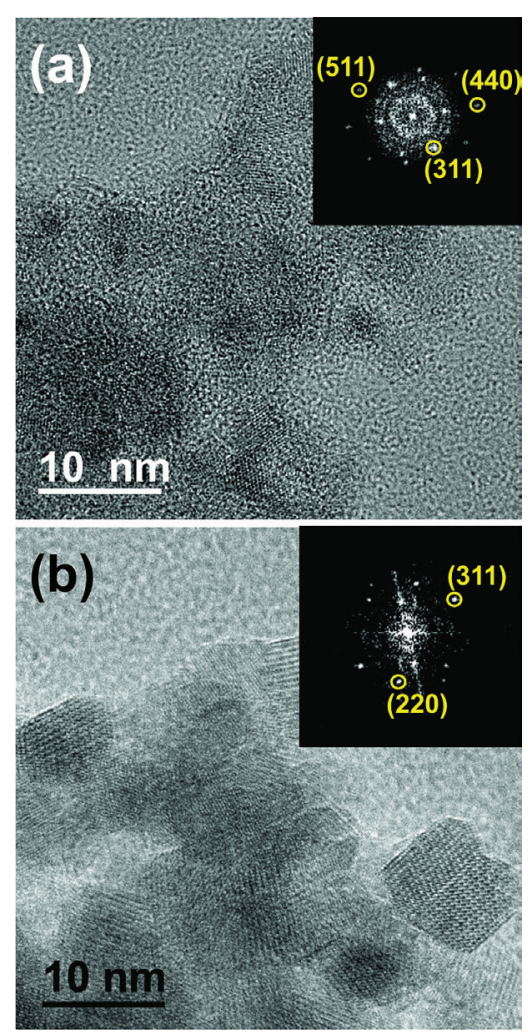

Figure 6. HRTEM images and corresponding FFTs of (a) S2-gly and (b) $\mathrm{S} 2-\varnothing$.

of the dissolution of the zinc ferrite mediated by glycerol and its derivatives.

Figure 8 shows the TEM and SEM images of the particles (with spherical shapes) produced by both routes

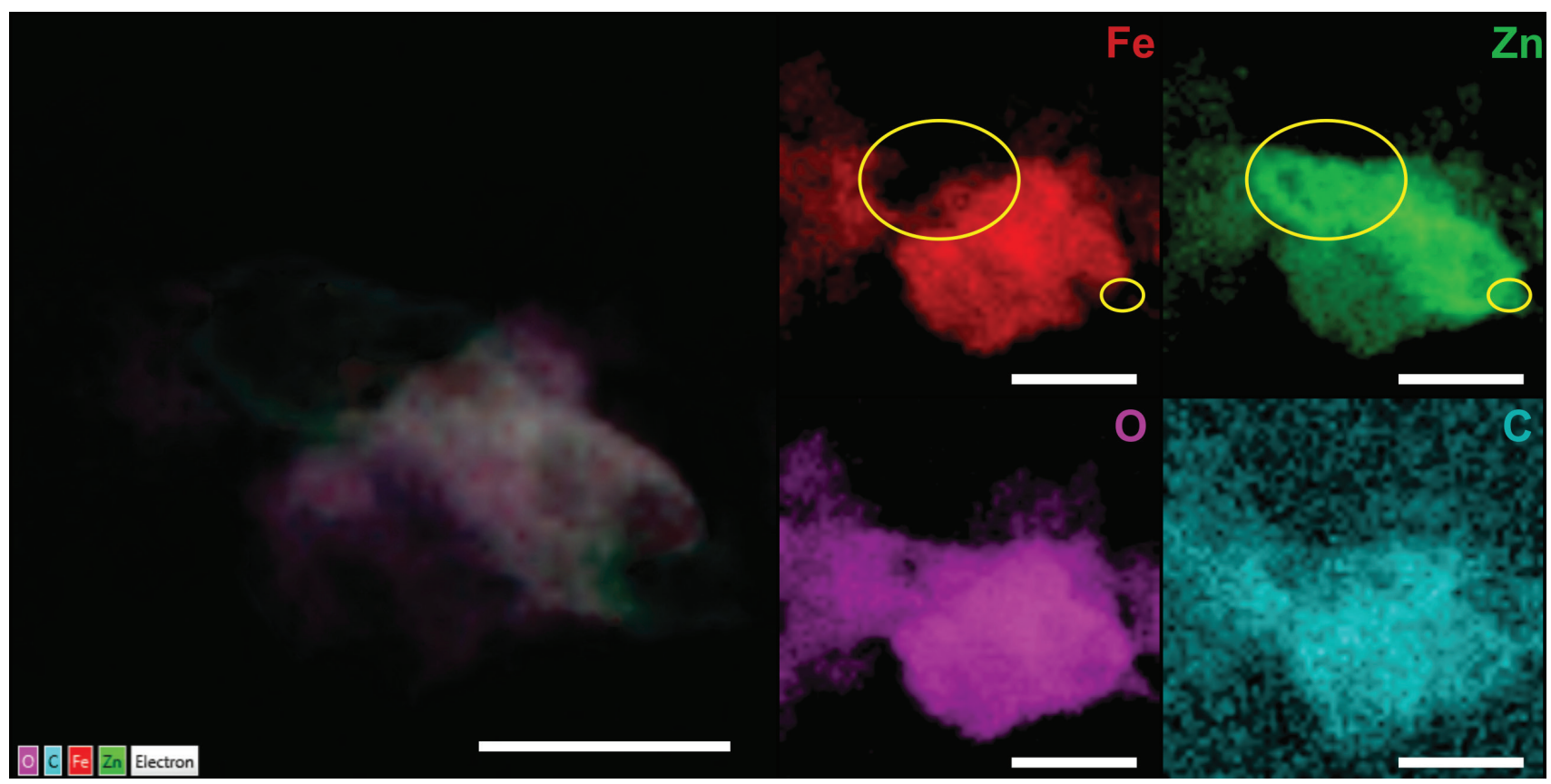

Figure 7. HAADF-STEM image and corresponding quantitative EDS maps for sample S2-gly (intensity proportional to atomic percentage). Iron (red), zinc (green), oxygen (magenta) and carbon (blue). The highlighted regions indicate zinc rich compound, consistent with the presence of zinc oxalate. Scale bar at $50 \mathrm{~nm}$. 
at the end of stage S3. However, the S3-gly sample presents particles which are still confined in the resin-like product, even after the thermal treatment applied (Figure 8a). These structures have dimensions in the range of hundreds nanometers (Figure 8c), which may contain a large number of particles (Figure 8b). Nevertheless, it is worth noting that the particles in these structures do not agglomerate, they remain dispersed in the organic structure preventing the aggregation and coalescence during this stage. Conversely, the sample S3- $\varnothing$ (Figure 8e) clearly shows an agglutination tendency of the particles.

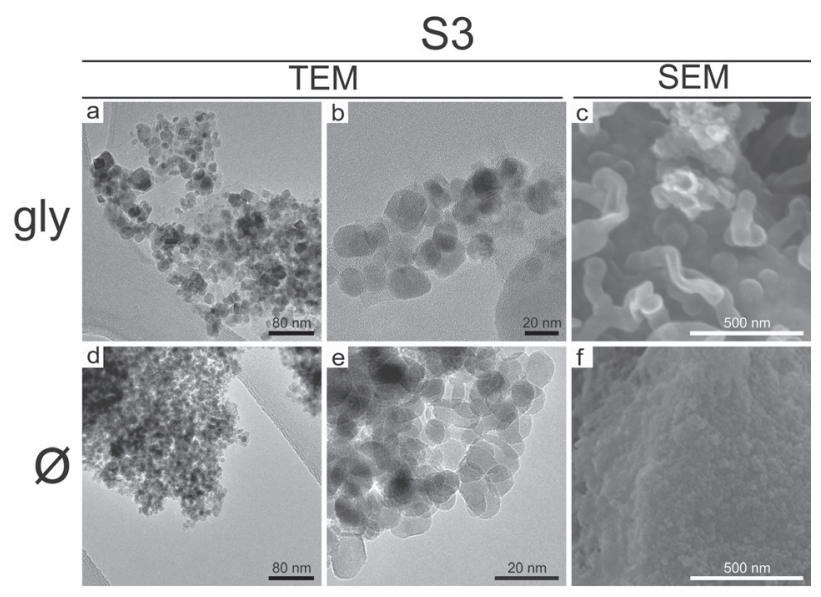

Figure 8. TEM and SEM micrographs show details of the morphology and aggregation of samples (a-c) S3-gly and (d-f) S3- $\varnothing$.

The differences between the samples produced by the modified and traditional methods become even more evident at the end of synthesis protocol (stage 4), as shown in Figure 9. As assessed by XRD, the crystals of the sample S4-gly are bigger, consequence of the dissolution and recrystallization mechanism provided by glycerol. Note that the glycerol also acts on the crystals morphology, leading to well-defined crystal facets and edges (Figure 9b). This is also related to the surface energy of the crystals in the nucleation and growth processes. For the sample $S 4-\varnothing$, the smaller size of the crystals is a direct result of the large number of nuclei formed during the precipitation, which limits the development of the crystals during the growth stage. ${ }^{25}$ The crystals obtained by the traditional method have less defined faces (Figure 9e) and show coalescence effect (Figure 9f). The size distribution histograms using a total of 250 particles for each sample S4-gly and S4- $\varnothing$ are shown in Figure S6 (SI section). As the S4-gly material has multiple facets, the length of the edges of these polyhedra was considered for the size measurement, whereas a spherical morphology was considered for the sample S4- $\varnothing$.

Although there is a large difference between the sizes

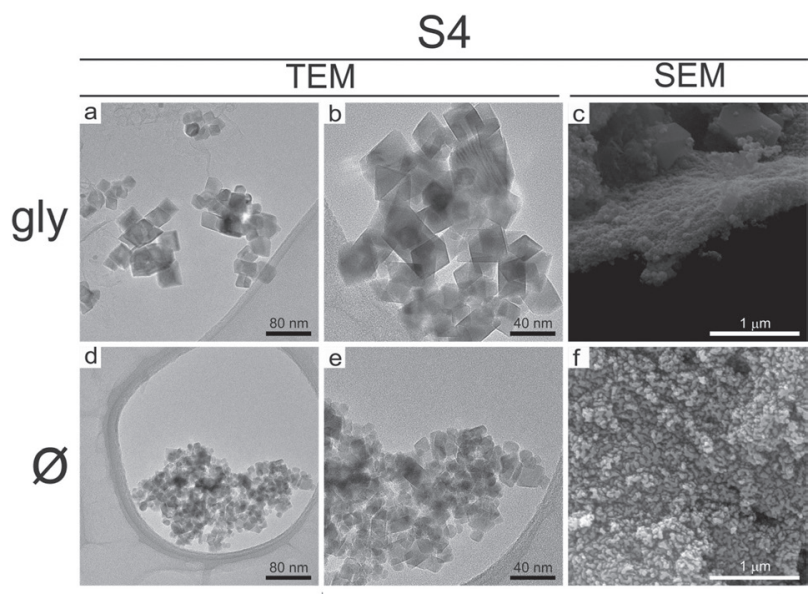

Figure 9. TEM and SEM micrographs of samples (a-c) S4-gly and (d-f) S4- $\varnothing$.

of the materials obtained by the different routes, both have a relatively narrow size distribution.

\section{TG/DTG}

Thermal analysis has been carried out as an auxiliary tool to better understand the composition of the different phases formed during the preparation of the zinc ferrite and, consequently, the mechanisms involved. The results of thermogravimetry are presented in Figure 10, and details concerning the thermal events are shown in Table 1. For the sake of comparison, the samples for each stage, with and without glycerol, are shown together, as well as their corresponding derivative curves. The solid line corresponds to the mass loss of the samples and the dotted line is the first derivative of the mass loss curves.

S1-gly and S1- $\varnothing$ samples (Figure 10a) present two and three well defined events, respectively. The first one is below $150{ }^{\circ} \mathrm{C}$, which is mostly related to the loss of physically adsorbed water from the structure of the samples. This is in agreement with mass spectra (MS) analysis (see Figure S7, SI section), confirmed by the presence of the molecular ion of water $\left(\mathrm{H}_{2} \mathrm{O}^{+}: m / z, 18\right)$ and fragment ions $\left(\mathrm{H}_{3} \mathrm{O}^{+}: \mathrm{m} / z, 19\right.$, $\mathrm{OH}^{+}: m / z, 17$ and $\left.\mathrm{O}^{+}: m / z, 16\right)$. The last events are more pronounced for sample S1-gly and reveal, rather than only the structural water loss observed in the sample S1- $\varnothing$, the presence of glycerol even after washing, indicating relevant interactions between glycerol and the material produced in the stage 1 , the mixed zinc ferrite. The MS data corroborate with this observation: peaks at $m / z, 12,13,22,27,31,39,43$ and 44 can be due to the release of the volatile compounds $\mathrm{C}^{+}, \mathrm{CH}^{+}, \mathrm{CO}_{2}{ }^{2+}, \mathrm{C}_{2} \mathrm{H}^{3+}, \mathrm{CH}_{2}=\mathrm{OH}^{+}, \mathrm{C}_{3} \mathrm{H}^{3+}, \mathrm{CH}_{2}=\mathrm{CH}-\mathrm{O}^{+}$and $\mathrm{CH}_{2}=\mathrm{CH}-\mathrm{OH}$, respectively, relative to the decomposition of the glycerol during the analysis. The presence of $\mathrm{m} / z 44$ in the sample without glycerol (S1- $\varnothing)$ can be associated 

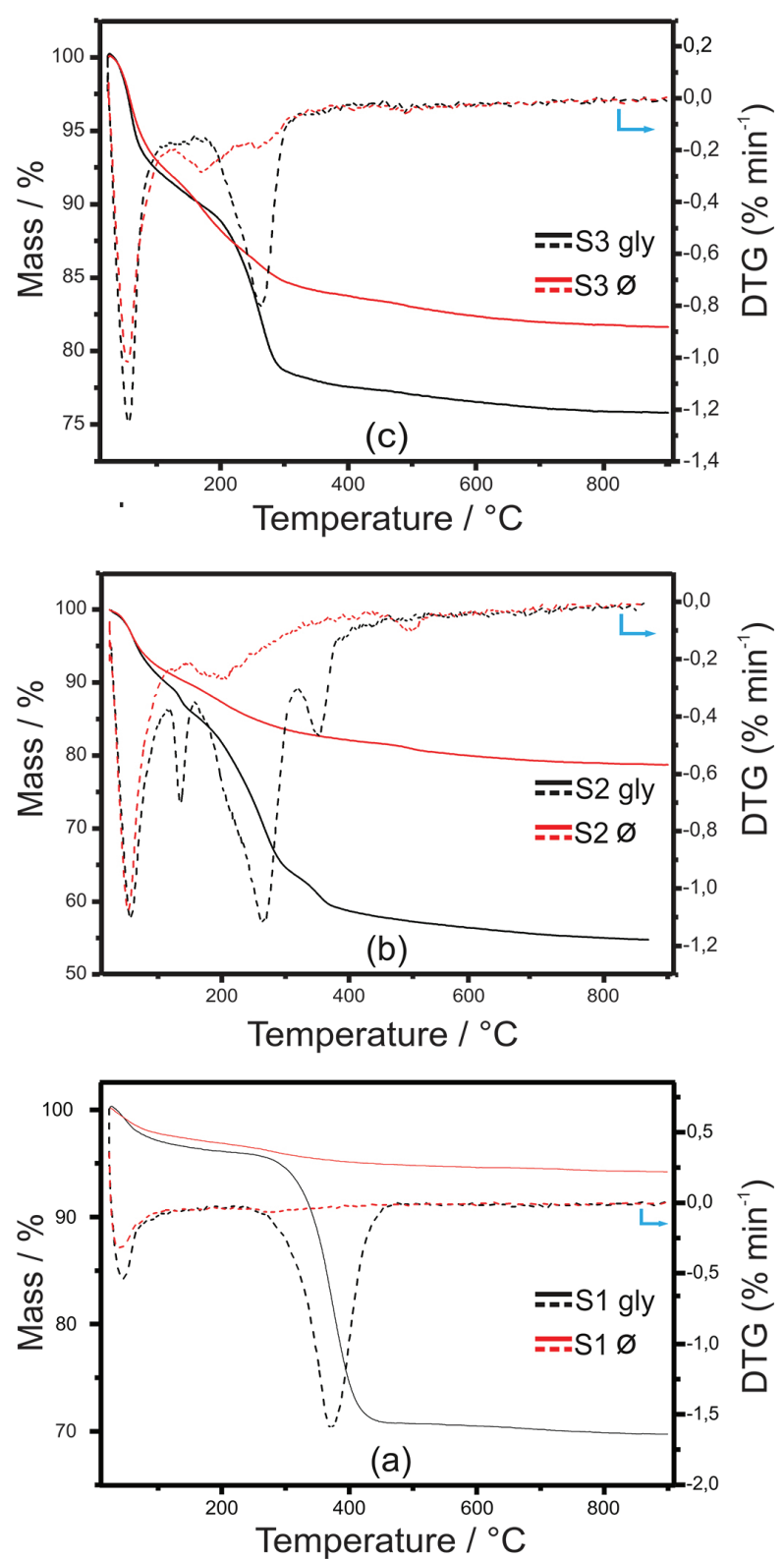

Figure 10. TG/DTG traces of samples (a) S1-gly and S1-Ø; (b) S2-gly and S2- $\varnothing$; and (c) S3-gly and S3- $\varnothing$. The solid line corresponds to the mass loss of the samples and the dotted line is the first derivative of the mass loss curves.

with the release of the $\mathrm{CO}_{2}$ adsorbed in the material due to the presence of zinc ferrite phase, as observed in the XDR. According to Hakim et al..$^{58} \mathrm{CO}_{2}$ can be adsorbed using oxides compounds and desorbed at high temperatures. This observation can also be used to explain the mass/charge ratio 44 for $\mathrm{S1-gly} \mathrm{sample.}$

The thermal profiles for S2 samples (Figure 10b) are relatively more complex. S2-gly presents four events. The first one is associated to water loss for both samples. In the case of S2-gly sample, the other events are related to the decomposition of glycerol-derived compounds, analogously to S1-gly sample, including the already
Table 1. Thermal analysis of the stages of zinc ferrite nanoparticles synthesis

\begin{tabular}{|c|c|c|c|c|c|}
\hline Sample & Event & $\begin{array}{c}\text { Initial } \\
\text { temperature / } \\
{ }^{\circ} \mathrm{C} \\
\end{array}$ & $\begin{array}{c}\text { Final } \\
\text { temperature / } \\
{ }^{\circ} \mathrm{C}\end{array}$ & $\begin{array}{c}\text { Lost } \\
\text { mass } / \%\end{array}$ & $\begin{array}{l}\text { Total lost } \\
\text { mass / \% }\end{array}$ \\
\hline \multirow{2}{*}{ S1-gly } & I & 24 & 145 & 9.24 & \multirow{2}{*}{21.67} \\
\hline & II & 145 & 321 & 12.43 & \\
\hline \multirow{3}{*}{ S1- $\varnothing$} & I & 24 & 120 & 7.90 & \multirow{3}{*}{15.40} \\
\hline & II & 120 & 242 & 5.50 & \\
\hline & III & 242 & 309 & 2.00 & \\
\hline \multirow{4}{*}{ S2-gly } & I & 27 & 117 & 10.36 & \multirow{4}{*}{40.78} \\
\hline & II & 117 & 156 & 3.90 & \\
\hline & III & 156 & 320 & 22.37 & \\
\hline & IV & 320 & 380 & 4.15 & \\
\hline \multirow{4}{*}{ S2- $\varnothing$} & I & 24 & 120 & 8.83 & \multirow{4}{*}{19.52} \\
\hline & II & 120 & 148 & 1.28 & \\
\hline & III & 148 & 436 & 8.15 & \\
\hline & IV & 436 & 531 & 1.26 & \\
\hline \multirow{2}{*}{ S3-gly } & I & 23 & 130 & 3.30 & \multirow{2}{*}{29.25} \\
\hline & II & 130 & 474 & 25.95 & \\
\hline \multirow{2}{*}{ S3- $\varnothing$} & I & 23 & 190 & 3.05 & \multirow{2}{*}{5.40} \\
\hline & II & 190 & 620 & 2.35 & \\
\hline
\end{tabular}

identified zinc oxalate. The thermal profile of $\mathbf{S 2}-\varnothing$, and the mass loss, is similar to $\mathrm{S} 1-\varnothing$. And, finally, samples $\mathrm{S} 3$ (Figure 10c) present two mass loss events. The initial loss is the same for the other samples: release of physically adsorbed water. The second one is much more intense for S3-gly and is consistent with the thermal degradation of the agglutinate, derived from glycerol, that involves ferrite particles, as observed in SEM and TEM images (Figure 8). These results are in good agreement with MS data (see Figure S7, SI section).

HPLC

Qualitative analysis was performed by HPLC to identify the products from the supposed oxidation of glycerol. The HPLC chromatograms of S1-gly and S2-gly samples are shown in Figure 11. As one can see, the sample S1-gly (Figure 11a) does not present any oxidation product, while the chromatogram for S2-gly shows the presence of glycerol oxidation products, including (1) oxalic, (2) glyceric, (3) glycolic and (4) formic acids (Figure 11b). The retention time data, doping process and the absorption spectra in the UV-Vis region were used to confirm these compounds. It is important to note that other products are also observed for the S2-gly sample, but they do not correspond to any of the standard samples used in the analysis. 


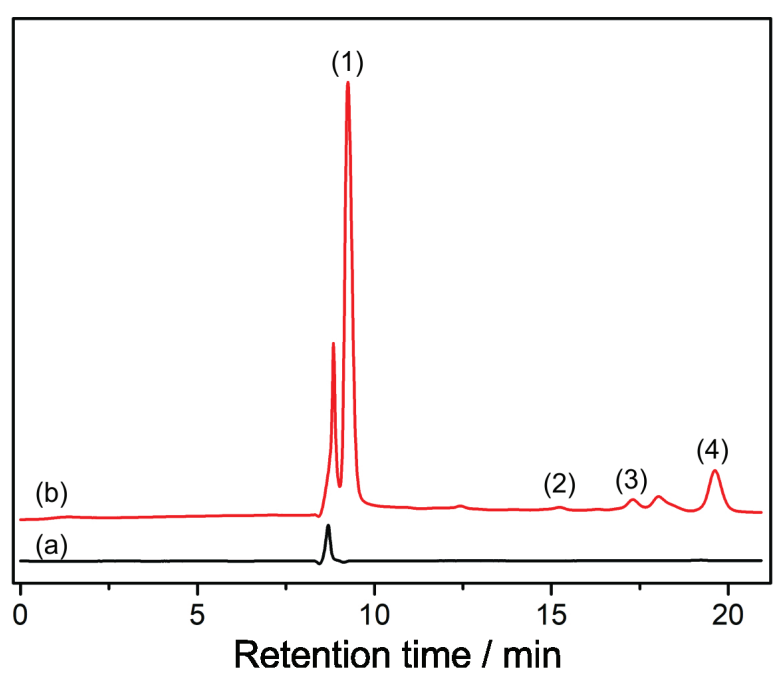

Figure 11. Chromatogram profiles of the samples at $210 \mathrm{~nm}$ for modified method: (a) S1-gly sample and (b) S2-gly sample. Label description of peaks: 1: oxalic acid; 2: glyceric acid; 3 : glycolic acid; 4: formic acid.

The large number of products at the end of stage S2 are derived from high-temperature effects, which provide the occurrence of parallel reactions. ${ }^{46,59}$ These compounds may have been reacted with the zinc ferrite, resulting in smaller crystals while zinc oxalate phase is produced, as pointed out both in XRD and TEM analyses.

\section{Conclusions}

This study has provided evidence of the role of glycerol as a stabilizing and structure directing agent for zinc ferrite nanoparticles synthesis by means of a modified coprecipitation method. Herein, we reported the modifications observed for the modified method in which the products generated by glycerol oxidation act on the zinc ferrite crystals synthesis process. The glycerol influence over the morphology of the zinc ferrite materials synthesized can be clearly observed when the crystals obtained from the different methods, the traditional and the modified ones, are compared.

The results of characterization carried out by the different techniques used in this work are consistent with a mechanism centered in partial dissolution and subsequent recrystallization of zinc ferrite mediated by glycerol and other compounds generated from it, which also act towards formation of intermediate phases and encapsulation of the formed crystals. The samples obtained by the coprecipitation modified method using glycerol showed better structural organization. Therefore, the modification of the coprecipitation method with glycerol induces to important improvements of physical properties of zinc ferrite nanoparticles produced, evidencing the potential of this method in the synthesis of ferrites.

\section{Supplementary Information}

Supplementary information is available free of charge at http://jbcs.sbq.org.br as PDF file.

\section{Acknowledgments}

The authors acknowledge Professor Luiz H. S. Gasparotto and MSc Thiago Y. B. Oliveira for invaluable discussions, Laboratório Nacional de Nanotecnologia (LNNano/CNPEM) for providing the equipment and technical support for the experiments involving TEM (ME-22646), Central Analítica of Universidade Federal do Ceará (UFC/CT-INFRA/MCTISISNANO/Pró-equipamentos-CAPES) for technical support and experiments of SEM, Brazilian Ministry of EducationCAPES and CNPq (process 402561/2007-4) for financial support.

\section{References}

1. Goodarz Naseri, M.; Saion, E. B.; Hashim, M.; Shaari, A. H.; Ahangar, H. A.; Solid State Commun. 2011, 151, 1031.

2. Guo, P.; Cui, L.; Wang, Y.; Lv, M.; Wang, B.; Zhao, X. S.; Langmuir 2013, 29, 8997.

3. Roonasi, P.; Nezhad, A. Y.; Mater. Chem. Phys. 2016, 172, 143.

4. Shahraki, R. R.; Ebrahim, S. A. S.; Masoudpanah, S. M.; J. Supercond. Novel Magn. 2015, 28, 2143.

5. Huang, X.; Zhang, J.; Xiao, S.; Sang, T.; Chen, G.; Mater. Lett. 2014, 124, 126.

6. Patil, S. B.; Bhojya Naik, H. S.; Nagaraju, G.; Viswanath, R.; Rashmi, S. K.; Vijay kumar, M.; Mater. Chem. Phys. 2018, 212, 351.

7. Anchieta, C. G.; Severo, E. C.; Rigo, C.; Mazutti, M. A.; Kuhn, R. C.; Muller, E. I.; Flores, E. M. M.; Moreira, R. F. P. M.; Foletto, E. L.; Mater. Chem. Phys. 2015, 160, 141.

8. Kant Sharma, R.; Ghose, R.; Ceram. Int. 2015, 41, 14684.

9. Choudhury, S.; Sinha, M.; Dutta, H.; Mandal, M. K.; Pradhan, S. K.; Meikap, A. K.; Mater. Res. Bull. 2014, 60, 446.

10. Kido, Y.; Nakanishi, K.; Kanamori, K.; RSCAdv. 2013, 3, 3361.

11. Bhosale, R. R.; Kumar, A.; AlMomani, F.; Alxneit, I.; Ceram. Int. 2016, 42, 2431.

12. Zhang, J.; Song, J.-M.; Niu, H.-L.; Mao, C.-J.; Zhang, S.-Y.; Shen, Y.-H.; Sens. Actuators, B 2015, 221, 55.

13. Xu, X.-J.; Zhou, L.-H.; Zhai, Q.-G.; Lu, C.-Z.; J. Am. Ceram. Soc. 2007, 90, 1959.

14. Yu, S.-H.; Fujino, T.; Yoshimura, M.; J. Magn. Magn. Mater. 2003, 256, 420.

15. Gharagozlou, M.; Bayati, R.; Superlattices Microstruct. 2015, 78, 190.

16. Chakraborty, M.; Thangavel, R.; Biswas, A.; Udayabhanu, G.; CrystEngComm 2016, 18, 3095. 
17. Nag, S.; Roychowdhury, A.; Das, D.; Das, S.; Mukherjee, S.; J. Magn. Magn. Mater. 2018, 466, 172.

18. Yadav, R. S.; Havlica, J.; Masilko, J.; Tkacz, J.; Kuřitka, I.; Vilcakova, J.; J. Mater. Sci.: Mater. Electron. 2016, 27, 5992.

19. Rishikeshi, S. N.; Joshi, S. S.; Temgire, M. K.; Bellare, J. R.; Znfe, S.; Dalton Trans. 2013, 42, 5430.

20. Nadeem, K.; Rahman, S.; Mumtaz, M.; Prog. Nat. Sci.: Mater. Int. 2015, 25, 111.

21. Tatarchuk, T. R.; Paliychuk, N. D.; Bououdina, M.; Al-Najar, B.; Pacia, M.; Macyk, W.; Shyichuk, A.; J. Alloys Compd. 2018, 731,1256

22. Raja, P.; Yadavalli, T.; Ravi, D.; Therese, H. A.; Ramasamy, C.; Hayakawa, Y.; Mater. Lett. 2017, 188, 406.

23. Vinosha, P. A.; Mely, L. A.; Jeronsia, J. E.; Krishnan, S.; Das, S. J.; Optik 2017, 134, 99.

24. Rani, B. J.; Ravi, G.; Yuvakkumar, R.; Ganesh, V.; Ravichandran, S.; Thambidurai, M.; Rajalakshmi, A. P.; Sakunthala, A.; Appl. Phys. A: Mater. Sci. Process. 2018, 124, 511.

25. Akbari, S.; Masoudpanah, S. M.; Mirkazemi, S. M.; Aliyan, N.; Ceram. Int. 2017, 43, 6263.

26. Ebrahimi, M.; Shahraki, R. R.; Ebrahimi, S. A. S.; Masoudpanah, S. M.; J. Supercond. Novel Magn. 2014, 27, 1587.

27. Majidi, S.; Zeinali Sehrig, F.; Farkhani, S. M.; Soleymani Goloujeh, M.; Akbarzadeh, A.; Artif. Cells, Nanomed., Biotechnol. 2014, 44, 722.

28. Darwish, M. S. A.; J. Mol. Liq. 2017, 231, 80.

29. Xia, T.; Wang, J.; Wu, C.; Meng, F.; Shi, Z.; Lian, J.; Feng, J.; Meng, J.; CrystEngComm 2012, 14, 5741.

30. Wang, S. F.; Li, Q.; Zu, X. T.; Xiang, X.; Liu, W.; Li, S.; J. Magn. Magn. Mater. 2016, 419, 464.

31. Grzyb, T.; Szczeszak, A.; Śniadecki, Z.; Idzikowski, B.; Lis, S.; J. Alloys Compd. 2016, 686, 489.

32. Jérôme, F.; Pouilloux, Y.; Barrault, J.; ChemSusChem 2008, 1, 586.

33. Larson, A. C.; Von Dreele, R. B.; General Structure Analysis System (GSAS); Los Alamos National Laboratory Report LAUR 86-748, Los Alamos, USA, 2004.

34. Toby, B. H.; J. Appl. Crystallogr. 2001, 34, 210.

35. Lopez, F. A.; Lopez-Delgado, A.; Martın De Vidales, J. L.; Vila, E.; J. Alloys Compd. 1998, 265, 291.

36. Giester, G.; Z. Kristallogr. 1997, 212, 720.

37. Heller, R. B.; Mcgannon, J.; Weber, A. H.; J. Appl. Phys. 1950, 21, 1283.

38. Scherrer, P.; Nachr. Ges. Wiss. Goettingen, Math.-Phys. Kl. 1918, $2,98$.
39. Schneider, C. A.; Rasband, W. S.; Eliceiri, K. W.; Nat. Methods 2012, 9, 671 .

40. Beltrán-Prieto, J. C.; Pecha, J.; Kašpárková, V.; Kolomazník, K.; J. Liq. Chromatogr. Relat. Technol. 2013, 36, 2758.

41. Jalalian, M.; Mirkazemi, S. M.; Alamolhoda, S.; J. Magn. Magn. Mater. 2016, 419, 363.

42. Huang, X.; Zhang, J.; Wang, W.; Sang, T.; Song, B.; Zhu, H.; Rao, W.; Wong, C.; J. Magn. Magn. Mater. 2016, 405, 36.

43. Aneesh Kumar, K. S.; Bhowmik, R. N.; Mater. Chem. Phys. 2014, 146, 159.

44. Kombaiah, K.; Vijaya, J. J.; Kennedy, L. J.; Bououdina, M.; Al-Lohedan, H. A.; Ramalingam, R. J.; Mater. Chem. Phys. 2017, 194, 153.

45. Kombaiah, K.; Vijaya, J. J.; Kennedy, L. J.; Kaviyarasu, K.; Mater. Chem. Phys. 2018, 221, 11.

46. Liu, S.-S.; Sun, K.-Q.; Xu, B.-Q.; ACS Catal. 2014, 4, 2226.

47. Sathyaseelan, V. S.; Rufus, A. L.; Subramanian, H.; Bhaskarapillai, A.; Wilson, S.; Narasimhan, S. V.; Velmurugan, S.; J. Nucl. Mater. 2011, 419, 39.

48. Musić, S.; Popović, S.; Maljković, M.; Dragčević, Đ.; J. Alloys Compd. 2002, 347, 324.

49. Hu, C.; Mi, J.; Shang, S.; Shangguan, J.; J. Therm. Anal. Calorim. 2014, 115, 1119.

50. Gnanaprakash, G.; Philip, J.; Jayakumar, T.; Raj, B.; J. Phys. Chem. B 2007, 111, 7978.

51. Parveen, R.; Tremiliosi-Filho, G.; RSC Adv. 2016, 6, 95210.

52. Cui, L.; Guo, P.; Zhang, G.; Li, Q.; Wang, R.; Zhou, M.; Ran, L.; Zhao, X. S.; Colloids Surf., A 2013, 423, 170.

53. Sundararajan, M.; John Kennedy, L.; Judith Vijaya, J.; J. Nanosci. Nanotechnol. 2015, 15, 6719.

54. Kant Sharma, R.; Ghose, R.; Ceram. Int. 2015, 41, 967.

55. Slatineanu, T.; Diana, E.; Nica, V.; Oancea, V.; Caltun, O. F.; Iordan, A. R.; Palamaru, M. N.; Cent. Eur. J. Chem. 2012, 10, 1799.

56. Waldron, R. D.; Phys. Rev. 1955, 99, 1727.

57. Pradeep, A.; Priyadharsini, P.; Chandrasekaran, G.; J. Alloys Compd. 2011, 509, 3917.

58. Hakim, A.; Marliza, T. S.; Tahari, N. M. A.; Isahak, R. W. N. W.; Yusop, R. M.; Hisham, W. M. M.; Yarmo, A. M.; Ind. Eng. Chem. Res. 2016, 55, 7888.

59. Farnetti, E.; Crotti, C.; Catal. Commun. 2016, 84, 1. 